Z. klin. Chem. u. klin. Biochem.

8. Jg., S. 51-55, Januar 1970

\title{
Ein neues Prinzip zur quantitativen Bestimmung hochmolekularer Antigene (Verknüpfungstest) und seine Anwendung auf Tetanustoxin, Serumalbumin und Ovalbumin
}

\author{
Von E. HabermanN \\ Pharmakologisches Institut der Justus Liebig-Universität Gießen \\ (Eingegangen am 21. August 1969)
}

Es wird ein neues, „Verknüpfungstest“ genanntes radioimmunologisches Verfahren zur quantitativen Bestimmung hochmolekularer Antigene beschrieben. Zunächst wird das zu bestimmende Antigen an einen Festphasen-Antikörper gebunden; dieses Antigen kann über seine noch freien determinanten Gruppen sckundär zugesetzte, markierte Antikörpermoleküle anlagern. Dadurch wird die Radioaktivität der gewaschenen Festphase ein Maß des Antigengehalts der ursprünglichen Lösung. Möglichkeiten und Grenzen des Verfahrens werden am Beispiel des Tetanustoxins demonstriert. Auch heterologes Serumalbumin und Ovalbumin lassen sich quantitativ in Gegenwart von Serum bestimmen. Die Nachweisgrenze für Ovalbumin und Tetanustoxin liegt unter $0,1 \mathrm{ng}$.

\section{$A$ newp radioimmunological procedure (“junction test") for the quantitative determination of bigh-molecular weight antigens applied to tetanus toxin, serum albumin and ovalbumin}

A new radioimmunological procedure ("junction test") for the quantitative determination of high-molecular weight antigens is described. The antigen to be determined is fixed by the corresponding solid-phase-antibody; then, labelled antibody is added which can be bound by the free determinant groups of the antigen. The radioactivity of the washed solid phase is correlated with the amount of antigen initially present as solute.

Potentialities and limits of the assay have been demonstrated with tetanus toxin. It is also useful for the determination of ovalbumin and heterologous serum albumin in the presence of serum. The detection limit for tetanus toxin and ovalbumin is below $0.1 \mathrm{ng}$.

Will man Pharmakodynamik und Pharmakokinetik hochmolekularer Naturstoffe, z. B. eiweißartiger Toxine, untersuchen, so benötigt man empfindliche und spezifische Nachweismethoden. Chemische Bestimmungsverfahren sind nur in Ausnahmefällen anwendbar, pharmakologische Wertbestimmungen erfordern viel Zeit und Tiermaterial. Beide erreichen oft nicht die gewünschte Empfindlichkeit und Genauigkeit. Selbst die heute bereits klassischen, auf Präzipitation beruhenden immunologischen Verfahren (z. B. in Agargel) sind für manche $\mathrm{Z}$ wecke nicht empfindlich genug.

Durch Verbindung immunologischer mit radiologischen Prozeduren läßt sich die Nachweisgrenze beträchtlich senken. Mehrere Kombinationen sind denkbar (Fig. 1). Als Beispiel eines Präzipitationsverfahrens auf radioimmunologischer Basis sei die früher beschriebene Radio-Retentionselektrophorese erwähnt, deren Empfindlichkeit durch die Löslichkeit der Immunkomplexe limitiert ist (1). Günstiger sind die kompetitiven Verfahren. Der Radioimmunassay (YALOW und Berson (2)) beruht auf der Kompetition markierten und unmarkierten Antigens um eine begrenzte Menge an Antikörper. Er hat sich bei der Bestimmung von Insulin, Glucagon und anderer, relativ niedermolekularer Antigene bewährt. Ein Festphasen-Radioimmunassay unter Verwendung kovalent an Cellulose gebundener Antikörper wurde von uns für Staphylokokken-Toxin erarbeitet (3). Ein auf dem nämlichen Prinzip beruhendes Verfahren wurde gleichzeitig und unabhängig für Immunglobulin beschrieben (4). Bei höhermolekularen Antigenen treten jedoch häufig Schwierigkeiten auf; so beobachteten wir eine „inverse" Reaktion beim Staphylokokken-Toxin
(3). Darüber hinaus wird der Test in der Regel nach Art eines subtraktiven Verfahrens ausgeführt, d. h. man ermittelt die Minderung der Radioaktivität des Immunkomplexes in Abhängigkeit von der Antigenkonzentration. Bei kleinen Antigenmengen beeinflußt also die Streuung des Ausgangswertes das Resultat sehr stark. Die Immunradiometrie (5) ist ebenfalls zu den kompetitiven Verfahren zu rechnen. Hierbei wird nicht das Antigen, sondern der Antikörper markiert. Er wird sich um so stärker an ein Festphasen-Antigen binden, je geringer der Gehalt an löslichem Antigen ist. Auch auf diese Prozedur treffen die Einwände gegen den klassischen Radioimmunassay zu.

Weder die auf Präzipitation noch die auf Kompetition beruhenden Methoden erfüllten also unsere Anforderungen bezüglich Empfindlichkeit, vor allem gegenüber hochmolekularen Antigenen. Daher mußte - abweichend von den bisherigen radioimmunologischen Prinzipien - eine neue Methode entwickelt werden, welche auf der Existenz mehrerer, räumlich getrennter Determinanten bei höhermolekularen Antigenen beruht.

\section{Material und Methoden}

Prinzip

Im ersten Schritt wird das Antigen an einen Antikörper adsorbiert, der an eine feste Phase, $z$. B. Cellulose, kovalent gebunden ist. Im zweiten Schritt werden die noch zugänglichen antigenen Determinanten mit markierten Antikörpern besetzt. Der ungebundene Anteil des Antikörperpräparates wird durch Waschen entfernt. Die Radioaktivität der Festphase steht in Beziehung zum Antigengehalt der ursprünglichen Lösung. Da markietter und FestphasenAntikörper über das Antigen verbunden werden, nennen wir den neuen Test ,Verknupfungstest". 


\section{Substanzen}

Festplosen-Antikörper wurden nach RoBbins und Mitarbeitern (6) durch kovalente Bindung an Cellulose hergestellt.

Festphasen-Tetanus-Antitoxin: 0,5 $\mathrm{ml}$ Tetanus-Antitoxin (FermoSerum vom Pferd, $3000 \mathrm{E} / \mathrm{m} l$; Behring-Werke, Marburg), $2 \mathrm{ml}$ $0,15 \mathrm{M}$ Phosphat-Citratpuffer $\mathrm{pH} 4,0$ und $4 \mathrm{ml}$ einer 2,64proz. wäßr. Suspension von Bromacetylcellulose (aus Cellulosepulver MN 300 HR, Macherey u. Nagel, Düren hergestellt) wurden gemischt und etwa $24 \mathrm{Stdn}$. bei Raumtemperatur gerührt. Weiter trurde wie bei (3) verfahren. Proteingehalt: $1,5 \mathrm{mg} / \mathrm{ml}(=5,7 \%$ der Trockensubstanz).

In analoger Weise ließ sich eine durch Ammoniumsulfatfällung hergestellte, dialysierte $\gamma$-Globulinfraktion antitoxischen Hammelserums (Handelspräparat der Behringwerke, Marburg) an Bromacetylcellulose binden. Proteingehalt $=3,0 \mathrm{mg} / \mathrm{ml}(=11,4 \%$ der Trockensubstanz).

Festphasen-Antiserumalbumin wurde durch Umsatz von $1 \mathrm{ml}$ $(4 \mathrm{mg} / \mathrm{ml}) \gamma$-Globulinfraktion eines gegen Humanalbumin immunisierten Kaninchens mit $2 \mathrm{~m} l$ Bromacetylcellulose-Suspension in Gegentwart.von $1 \mathrm{~m} l$ des genannten Puffers hergestellt $(0,11 \mathrm{mg}$ Protein/mg Trockensubstanz).

Festpbasen-Antiovalbumin: Durch Immunisierung von Meerschweinchen mit Ovalbumin in FREUndschem Adjuvans wurde ein Antiserum gewonnen, aus dem durch Ammoniumsulfatfällung die $\gamma$-Globulinfraktion hergestellt wurde. Diese wurde analog wie Anti-Serumalbumin an Bromacetylcellulose gekoppelt.

\section{Natives Antigen}

Hochgereinigtes 'Tetanustoxin verdanken wir Herrn Dr. A. Turprn, Institut Pasteur, Paris. Das Präparat enthielt $24 \mathrm{mg}$ Protein $/ \mathrm{m} l$, welche $12000 \mathrm{Lf}^{1}$ ) entsprachen. Die subkutaneLD 50 (Maus) lag zwischen 2,5 und 1,25 ng/kg, was $2-4 \times 10^{7} \mathrm{LD}$ $50 / \mathrm{mg}$ Protein entspricht. Die höchsten bisher in der Literatur angegebenen Werte liegen bei $10^{8}$ Mäuse-LD/mg Stickstoff. Das von uns benutzte Toxinpräparat ist also voll aktiv.

Menschliches Serumalbumin, hochgereinigt (Behringwerke Marburg). Ovalbumin: $5 \times$ krist., $>99 \%$ rein (Serva-Entwicklungslabor, Heidelberg).

\section{Markiertes Antigen}

Umsatz hochgereinigten Tetanustoxins mit ${ }^{125} \mathrm{~J}$ bzw. ${ }^{131} \mathrm{~J}$ nach (7); Nachreinigung an Sephadex G 200. Es wurden Präparate mit einer spezifischen Radioaktivität von 19 bzw. $10 \mathrm{mC} / \mathrm{mg}$ erhalten. Die markierten Tetanustoxinpräparate bestanden zu $80-82 \%$ aus immunologisch aktivem Material. Die Toxizität geht unter diesen Bedingungen zu etwa $75 \%$ verloren, und zwar wegen des Kontaktes mit Chloramin T.

\section{Markierte $\gamma$-Globulinfraktionen}

$\mathrm{Da}$ wir mit immunologisch einheitlichen Antigenen arbeiteten, wäre eine Reinigung der zu ihrem Nachweis benötigten Antikörper prinzipiell nicht erforderlich gewesen. In der Tat ließ sich durch direkte Markierung eines $\gamma$-Globulinpräparates aus Hammelserum ein Reagenz mit $2 \mathrm{mC}{ }^{131} \mathrm{~J} / \mathrm{mg}$ Protein erhalten, das sich zu etwa $10 \%$ an Festphasen-Toxin binden ließ und für den Verknüpfungstest ebensogut brauchbar war wie das im folgenden beschriebene gereinigte Präparat.

Wir gingen dabei - wie Mrles und Hales (5) - von der Vorstellung aus, daß die antideterminanten Gruppen des Antikörpers bei der Jodierung geschützt werden müßten. Daher stellten wir ein Festphasen-Toxin her, indem wir unter den beschriebenen Standard-Bedingungen $5 \mathrm{~m} l$ dialysiertes Tetanus-Rohtoxin (Behringwerke, $1,2 \mathrm{mg} / \mathrm{ml}$ ) in Gegenwart von $2 \mathrm{~m} l$ Puffer mit $4 \mathrm{ml}$ Bromacetylcellulose-Suspension umsetzten. Das Endprodukt wurde in $3 \mathrm{~m} l \mathrm{H}_{2} \mathrm{O}$ aufgenommen. $0,1 \mathrm{~m} l$ dieses Immunsorbens wurden mit $0,1 \mathrm{~m} /$ Antitoxin (Fermo-Serum, Behringwerke) $1 \mathrm{Std}$. bei $0^{\circ}$ geschüttelt, abzentrifugiert, $4 \mathrm{mal}$ mit $0,15 \mathrm{M} \mathrm{NaCl}$ gewaschen und in $200 \mu l 0,05 \mathrm{M}$ Phosphatpuffer $\mathrm{pH} \mathrm{7,2} \mathrm{aufgenommen.} \mathrm{Der}$ Immunkomplex wurde nunmehr jodiert, indem $200 \mu l 0,05 \mathrm{M}$

1) $1 \mathrm{Lf}=$ diejenige Antigenmenge, welche mit 1 Einheit Antitoxin optimal präzipitiert.
Puffer mit $260 \mu \mathrm{C}{ }^{125} \mathrm{~J}$ und $100 \mu l$ 0,1proz. Chloramin T zugesetzt wurden; $1 \mathrm{Min}$. später wurde mit $50 \mu l(240 \mu \mathrm{g}) \mathrm{Na}_{2} \mathrm{~S}_{2} \mathrm{O}_{5}$ abgestoppt. Nach Zentrifugieren wurde $5 \mathrm{mal}$ mit einer Lösung von $0,15 \mathrm{M} \mathrm{NaCl}$ und $0,01 \mathrm{M} \mathrm{NaJ}$ gewaschen. Der Antigen-Antikörperkomplex wurde durch einstündiges Schütteln in $0,1 \mathrm{M}$ Essigsäure bei $37^{\circ}$ dissoziiert, zentrifugiert und neutralisiert. Von der insgesamt eluierbaren Radioaktivität befanden sich $29 \%$ im Essigsäure-Extrakt; davon.wiederum waren etwa $80 \%$ immunreaktiv, d. h. an Festphasen-Toxin erneut zu binden.

Markiertes Anti-Humanalbumin wurdé durch Jodierung (in Lösung) der $\gamma$-Globulinfraktion von Antiserum (Kaninchen) hergestellt $\left(1,3 \mathrm{mC}{ }^{131} \mathrm{~J} / \mathrm{mg}\right.$ Protein). Nach Gelfiltration an Sephadex G 200 erwiesen sich $21 \%$ des Präparates als bindungsfähig an Festphasen-Albumin (nach RosBINS und Mitarbeiter (6) hergestellt).

In analoger Weise gewannen wir markiertes Anti-Ovalbumin. Es wurden $1,5 \mathrm{mC}{ }^{125} \mathrm{~J} / \mathrm{mg}$ Protein gebunden. Etwa $35 \%$ des Präparates konnten eine Bindung mit Festphasen-Ovalbumin eingehen.

\section{Versuche zur Methodik}

Vergleich von Verknïpfungstest mit Festphasen-Radioimmunassay und Immunradiometrie

Abbildung 2 gibt die Dosis-Wirkungsbeziehung im Verknüpfungstest für Tetanustoxin, Serumalbumin und Ovalbumin wieder. Die Nachweisgrenze liegt für Tetanustoxin und Ovalbumin um 0,1 ng/Ansatz, für Serumalbumin etwa 10 mal höher. In keinem Fall ist die Dosis-

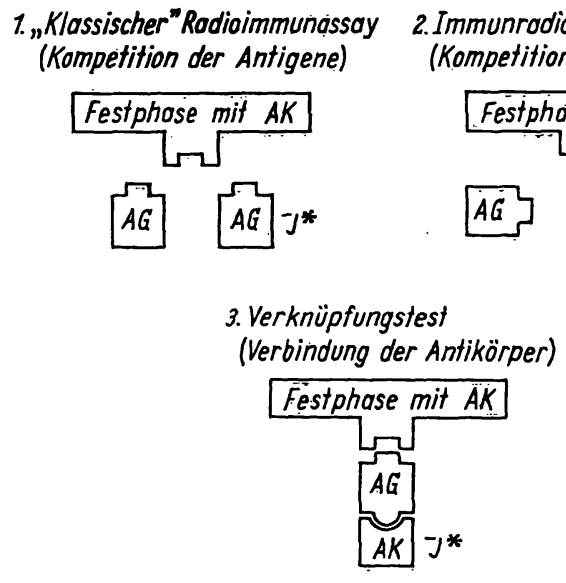

Abb. 1

Prinzipien radioimmunologischer Bestimmungsmethoden

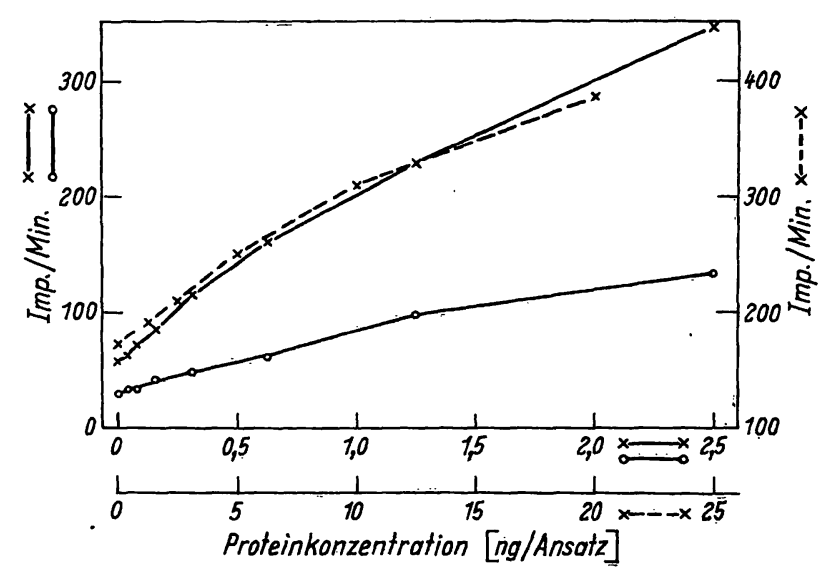

Abb. 2

Beziehung zwischen vorgelegter Menge an Tetanustoxin, Serumalbumin bzw. Ovalbumin und Antikörperbindung. Bedingungen: s. S. 54 (Standardansatz). Ordinate: Radioaktivitä̈t der Festphase (Imp./Min.) bei Einsàtz von Tetanustoxin $(x-x)$ oder Ovalbumin $(0-0)$ (links); Serumalbumin $(x--x)$ (rechts). Abszisse: ng Tetanustoxin bzw. 'Ovalbumin/Ansatz (oben); 'ng Serumalbumin/Ansatz 
Wirkungs-Beziehung streng linear, so daß stets Eichreihen zur Interpolation der unbekannten Antigenlösung erforderlich sind. Die mittlere Abweichung aller in Abbildung 2 eingezeichneten Mittelwerte liegt für Tetanustoxin bei $S_{\overrightarrow{\mathbf{x}}}= \pm 2,14 \%$.

Der Verknüpfungstest ist dem Solid-phase-Radioimmunassay und der Immunradiometrie deutlich überlegen. Die Empfindlichkeitsgrenze für Tetanustoxin liegt beim Radioimmunassay bei $5 \mathrm{ng} /$ Ansatz (Abb. 3). Es tritt das

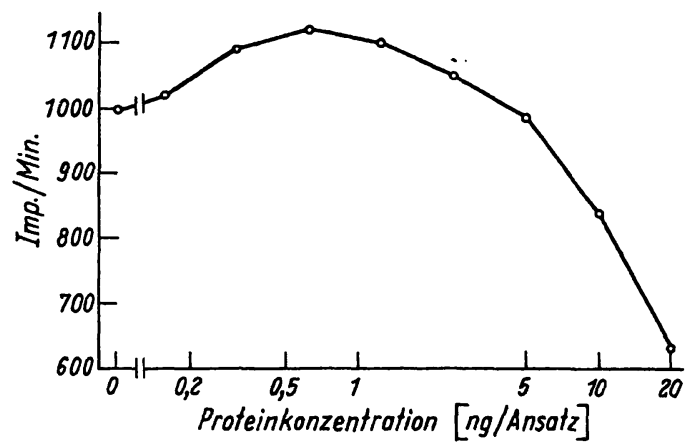

Abb. 3

Festphasen-Radioimmunassay von Tetanustoxin Ansätze: $0,1 \mathrm{ml}$ Kaninchenserum + 0,05 $\mathrm{ml} 0,1 \mathrm{M} \mathrm{Na} \mathrm{N}_{2}$ EDTA (pH 7,5) $+0,1 \mathrm{ml}$ Tetanus-Toxin-Verdünnung in gepufferter Albuminlösung
$+0,1 \mathrm{ml}$ markiertes (2801 Imp./Min.) Tetanustoxin $+0,1 \mathrm{ml}$ Festphasen-Antitoxin (entsprechend 13,2 $\mu \mathrm{g}$ Trockensubstanz); über Nacht bei Raumtemperatur rotiert; Festphase 2 mal gewaschen. Ordinate: Radioaktivität der Festphase (Imp./Min.). Abszisse: ng

Phänomen des „inversen Radioimmunassay“ (3) in Erscheinung, wodurch die Bestimmung erheblich gestört wird: Die Bindungskurve durchläuft ein Maximum, das über dem Kontrollwert liegt. Die Immunradiometrie stand in ihrer Empfindlichkeit für Tetanustoxin zwischen dem Festphasen-Radioimmunassay und dem Verknüpfungstest.

\section{Einfluß einiger Versuchsbedingungen auf den Verknüpfungs-} test

Es ist darauf zu achten, daß nicht etwa Mischformen $z$ wischen kompetitiven und Verknüpfungsverfahren auftreten. Daher sollte das zu bestimmende Antigen vollständig absorbiert sein, ehe der markierte Antikörper

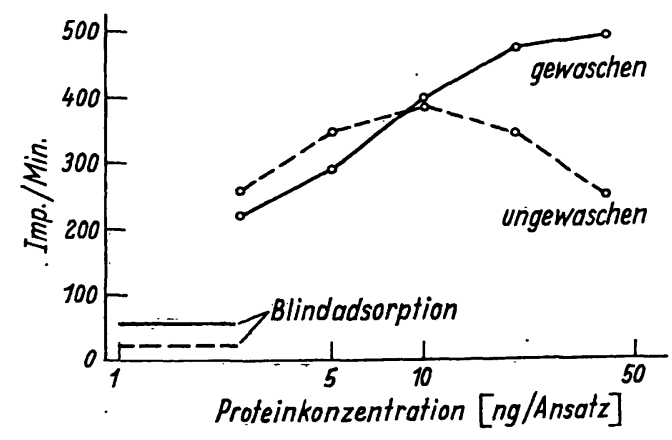

Abb. 4

Hemmung des Verknüpfungstests durch Antigenüberschuß (0- - - o) und seine Normalisierung durch Waschen $(-0)$ Ordinate: Radioaktivität (Imp./Min.) der Festphase. Abszisse : ng wet inten unter StandardAnsatz. Die "ungewaschenen "Festphasen wurden unter Standach der ersten bedingungen (s. S. 54) erhalten, da gewaschit gepufferter Albuminlösung von überschússigem Antigen befreit zugesetzt wird. Bei hohen Antigenkonzentrationen reicht die Menge des eingesetzten Immunsorbens nicht aus, so daß freies Antigen den markierten Antikörper in Lösung hält (Abb. 4). Dieser Schwierigkeit könnte man am einfachsten dadurch entgehen, da $B$ man von vornherein eine größere Menge Immunsorbens zusetzt. Man nimmt dabei eine verstärkte unspezifische Bindung des markierten Antikörpers an die Festphase in Kauf. Besser wäscht man das Immunsorbens nach der Bindung des Antigens, ehe man den markierten Antikörper zusetzt. Dabei nimmt allerdings die Empfindlichkeit etwas ab. $\mathrm{Da}$ die Umkehr der Kurve unter unseren Bedingungen erst beim Mehrhundertfachen der Nachweisgrenze auftritt, haben wir die zwischenzeitliche $\mathrm{X}$ /aschung nicht in unser Standardverfahren aufgenommen. - Ein analoges Phänomen (Abnahme der gebundenen Radioaktivität oberhalb eines Antigengehalts um $100 \mathrm{ng} /$ Ansatz) fand sich auch bei Verwendung von Humanalbumin und Ovalbumin.

Der Verknüpfungstest sollte in erster Linie der Bestimmung von Antigenen im Blutplasma dienen. Daher war es wichtig zu wissen, ob Plasma die spezifische oder unspezifische Bindung markierten Antitoxins beeinflußt. In Abwesenheit von Plasma oder Serum wurde, wie Abbildung 5 zeigt und die Versuche mit Serumalbumin

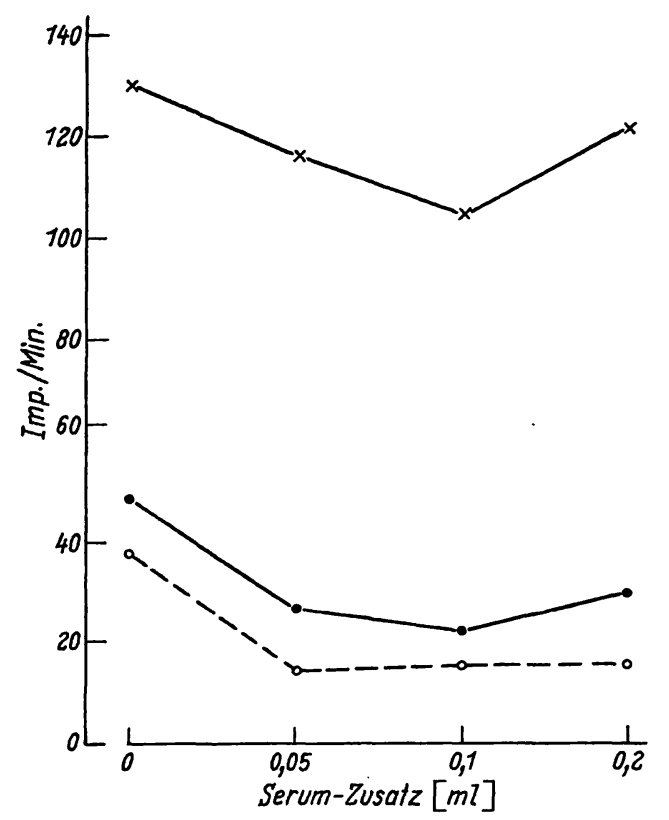

Abb. 5

Unspezifische Bindung des markierten Antikörpers in Abhängigkeit von der Serummenge/Ansatz

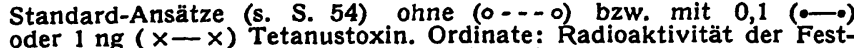
phase (Imp./Min.). Abszisse: $\mathrm{ml}$ Kaninchenserum/Ansatz

und Ovalbumin bestätigten, mehr markiertes Antitoxin unspezifisch gebunden. Die Eichreihe sollte stets mit derjenigen Plasmakonzentration hergestellt werden, die auch in den Versuchsansätzen vorliegt.

Die Inkubationszeit geht in unser System zweifach ein: bei der Reaktion zwischen Antigen und FestphasenAntikörper und bei der Reaktion zwischen dem so ent- 
standenen Komplex und dem markierten Antikörper. Die Bindung des Antigens ist in den ersten zwei Stunden nahezu vollständig; wir wählten vier Stunden für die

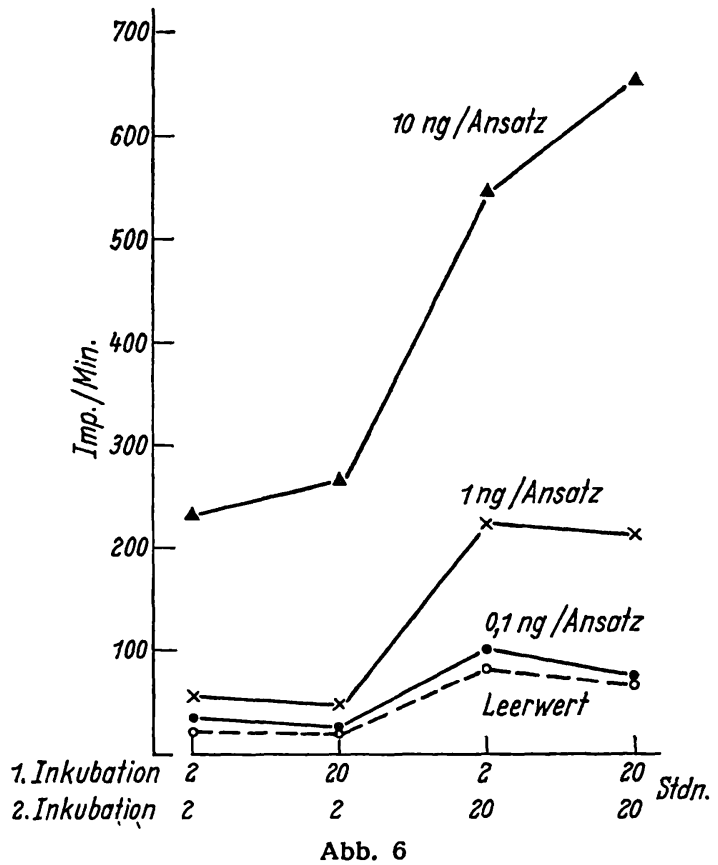

Abhängigkeit der Bindung des markierten Antikörpers von der Inkubationszeit

Ordinate: Radioaktivität der Festphase (Imp./Min.) nach Inkubation mit Tetanustoxin (-. $0,1 \mathrm{ng} / \mathrm{Ansatz} ; \times-\times 1,0 \mathrm{ng} / \mathrm{Ansatz} ;$ $10 \mathrm{ng} /$ Ansatz) bzw. gepufferter Albuminlösung (0-- - $)$. Ábszisse der zweiten Inkubation (Zusatz von markiertem Antitoxin). Im übrigen: Standardbedingungen (s. S. 54)

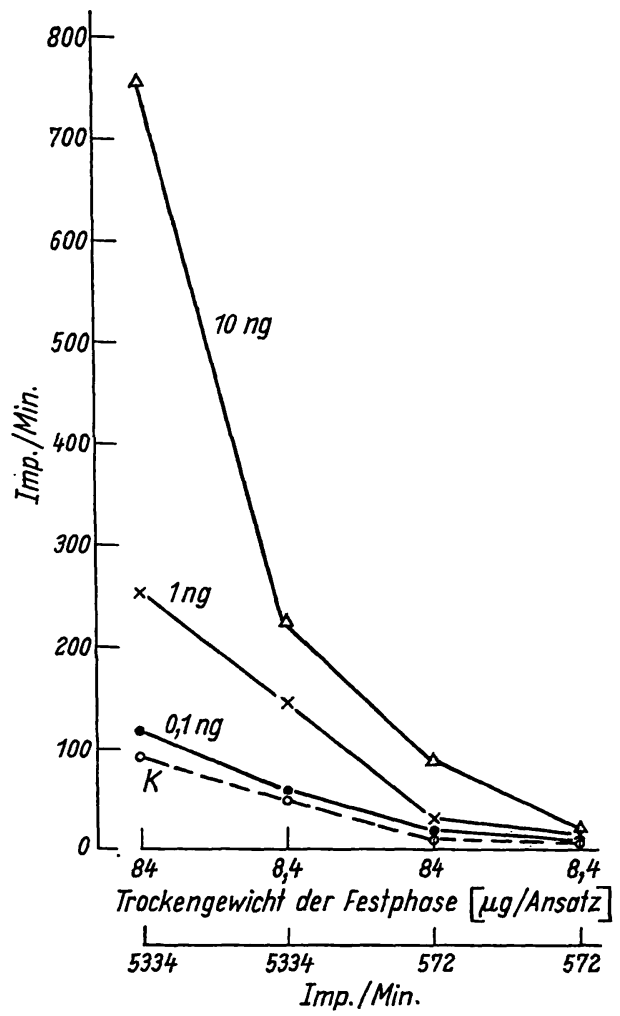

Abb. 7

Einfluß der Menge des markierten Antikörpers und der Festphase. Ordinate: Radioaktivität (Imp./Min.) der Festphase bei Einsatz von Tetanustoxin $(-)$ bzw. phosphatgepufferter Albuminlösung $(---)$. Absisse: Trockengen der zugesetzten Festphase ( $\mu \mathrm{g})$; RadioStandardbedingungen (s. S. 54) erste Inkubation des Standard-Ansatzes. Dagegen benötigt die Bindung des markierten Antikörpers längere Zeit. Möglicherweise sind auch 20 Stunden noch nicht ausreichend, um den Endpunkt der Reaktion zu erreichen. Doch dehnten wir die Zeit nicht weiter aus, weil mit der Dauer der zweiten Inkubation auch die unspezifische Adsorption zunahm und den Nachweis kleiner ( $<1 \mathrm{ng} /$ Ansatz) Mengen, störte (Abb. 6).

Als letzte Variante war die Bedeutung der Konzentration von markierten und von Festphasen-Antikörpern $\mathrm{zu}$ studieren. Theoretisch sollten beide Reagenzien in beliebigem Úberschuß verwendbar sein; die Radioaktivität der festen Phase sollte allein von der Menge des gebundenen Antigens abhängen. In praxi steht dem eine unspezifische Bindung des markierten Antikörpers entgegen; sie begrenzt den Zusatz beider Reagenzien. Die Beziehungen sind in Abbildung 7 dargestellt. Zusatz noch größerer Mengen an Festphase brachte keine vermehrte spezifische Bindung des markierten Antikörpers über das Antigen mit sich, nur noch eine Erhöhung des Leerwertes. Es fällt auf, daß nur ein Teil der Radioaktivität des Antitoxinpräparates an die Festphase gebunden wird.

\section{Standard-Ansatz}

Unter Berücksichtigung der besprochenen Variablen ergibt sich folgender Standardansatz für die Bestimmung höhermolekularer Antigene:

$0,2 \mathrm{ml}$ antigenhaltiges $(>0,1 \mathrm{ng}$ Tetanustoxin bzw. Ovalbumin bzw. $<1$ ng Humanalbumin) Serum bzw. Kontrollserum werden mit

$0,1 \mathrm{ml} 0,1 \mathrm{M} \mathrm{Na} \mathrm{NDDTA}_{2} \mathrm{pH} 7,5$ (zur Inaktivierung von Komplement) und

$0,1 \mathrm{ml}$ phosphatgepufferter $(0,05 \mathrm{M}, \mathrm{pH} 7,5) \quad 0,85$ proz. $\mathrm{NaCl}-L o ̈ s u n g ~ m i t ~ 0,1 \%$ Rinderserumalbumin als Stabilisator versetzt. In der Albumin-NaCl-Lösung wird das Antigen der Kontrollreihen verdünnt. Nach Umschütteln setzt man

$0,1 \mathrm{~m} l$ einer (zuvor titrierten) Verdünnung von frisch gewaschener, gründlich zerteilter Suspension (Magnetrührer!) von Festphasen-Antikörper zu. Man möchte Verluste durch Adsorption an Pipetten usw. vermeiden und das Präzipitat nach dem Zentrifugieren erkennen; deshalb werden die Verdünnungen in 0,01 proz. Suspension von MN 300-Cellulose hergestellt. Bei Raumtemperatur wird etwa 4 Stdn. mit etwa 14 U./Min. um die Querachse rotiert (1. Inkubation), dann der markierte Antikörper zugesetzt (etwa $5000 \mathrm{Imp}$./Min.) und erneut etwa $16 \mathrm{Stdn}$. rotiert (2. Inkubation). Nun wird abzentrifugiert (2 Min.; Eppendorf-Zentrifuge 3200), das Uberstehende abgesaugt, der Bodensatz mit einem Beckman-Micromixer (Nr. 154) in phosphatgepufferter Albuminlösung suspendiert, zentrifugiert und die Waschung wiederholt. Weitere Waschungen brachten keine Radioaktivität mehr in Lösung. Die Radioaktivität des Bodensatzes wird im Autogamma-Spektrometer (Packard) bestimmt. 


\section{Diskussion}

Das neue Verfahren wurde in erster Linie für Tetanustoxin und verwandte Substanzen ausgearbeitet; es ist jedoch, wie die Versuche mit Humanalbumin und Ovalbumin zeigen, auch für andere höhermolekulare Antigene brauchbar. Wahrscheinlich sind auf diesem Weg alle Antigene bestimmbar, welche mehrere Determinanten tragen und daher, falls sie in höherer Konzentration vorliegen, mit Antikörpern präzipitieren. Es erscheint nicht ausgeschlossen, daß auf einem einzigen Antigenmolekül mehrere markierte Antikörpermoleküle Platz finden. Eine weitere Steigerung der Empfindlichkeit könnte vielleicht dadurch erzielt werden, daß die Antikörpermoleküle nicht markiert, sondern, entspre- chend einem Vorschlag von Miles und Hales (5), mit einem gut nachweisbaren Enzym gekoppelt werden. Aus unserer eingangs dargestellten Vorstellung über den Reaktionsablauf ergibt sich, daß der Verknüpfungstest kaum den Radioimmunassay relativ niedermolekularer Antigene ersetzen wird. Der neue Test ist eher bei großen Antigenen, auch in der Virologie, der Prüfung wert. Gerade hier treten experimentelle, theoretisch begründbare Schwierigkeiten mit dem klassischen Radioimmunassay auf.

Für die Unterstützung dieser Untersuchungen bin ich der Deutschen Forschungsgemeinschaft sowie dem Bundesinnenministerium zu großem Dank verpflichtet. Herrn Dr. RäkER verdanke ich die markierten Proteine. Fräulein ZEUNER leistete geschickte technische Assistenz.

\section{Literatur}

1. Habermann, E., K. O. Räker und D. Arndts, Klin. Wschr. 46, 46 (1968). - 2. Yalow, R. und S. A. Berson, Nature London 184, 1648 (1959). - 3. HABERMANN, E., K. O. RÄker und G. Zeuner, Naunyn-Schmiedebergs Arch. Pharmak. exp. Path. 262, 165 (1969). 4. MANN, D., H. Granger und J. L. Fafey, J. Im- munol. Baltimore 102, 618 (1969). - 5. MrLEs, L. E. M. und C. N. Hales, Lancet London 1968/II, 492. - 6. RobBins, J. B., J. HaIMOVICH und M. SELA, Immunochemistry 4, 11 (1967). - 7. Greenwood, F. C., W. M. Hunter und J. S. Glover, Biochem. J. 89, 114 (1963).
Prof. Dr. E. Habermann Pharmakologisches Institut der Justus-Liebig-Universität 63 Gießen

Rudolf-Buchheim-Str. 4 